\title{
Leminorella richardii
}

National Cancer Institute

\section{Source}

National Cancer Institute. Leminorella richardii. NCI Thesaurus. Code C86494.

A species of facultatively anaerobic, Gram negative, rod shaped bacteria in the phylum Proteobacteria. This species is hydrogen sulfide positive, citrate and oxidase negative and produces acid from $L$ arabinose and $D$ xylose but not dulcitol. L. richardii is an emerging nosocomial pathogen, especially in immunocompromised patients. 